\title{
Integrated Instrumentation Amplifier for the Phase Readout of Piezoresistive Strain Gauges
}

\author{
Reinoud F. Wolffenbuttel and Arnold R. Schekkerman
}

\begin{abstract}
An integrated instrumentation amplifier combined with a bandpass filter has been fabricated in a bipolar process for the phase readout of piezoresistive sensors. Changes in the resistance can be measured directly using an ac-operated bridge configuration. A special technique has been employed to convert these changes into a phase angle relative to the excitation signal. The instrumentation amplifier should have a very high Common-Mode Rejection Ratio (CMRR) at a relatively high bridge operating frequency $(100 \mathrm{kHz})$ in order to actually exploit the advantages of this readout method. The filter should have a well-determined phase behavior to prevent a change in the frequency from affecting the output phase angle. Special emphasis has been placed on the compatibility between micromachining technologies in silicon and bipolar processing, so that a fully integrated smart silicon micromechanical sensor can be made.
\end{abstract}

\section{INTRODUCTION}

$\mathbf{P}$ IEZORESISTIVE strain gauges find widespread application in stress measurement in mechanical structures. These devices are customarily used in both engineering constructions and micromechanical silicon sensors [1]. Although capacitive techniques are rapidly emerging, the robustness and simplicity in operation of the piezoresistive strain gauge will ensure the prolongation of its position as factual industrial standard. The temperature sensitivity is compensated using the Wheatstone bridge configuration with all four resistors attached to the thermally conducting (micro)mechanical structure. The piezoresistors are placed in such a way that two are subjected to tensile stress, and two are unloaded or experience compressive stress.

The bridge is usually ac-operated to prevent offset in the readout circuitry from increasing the threshold of detectivity. A coherent detector is subsequently employed for conversion of the bridge imbalance-induced ac amplitude variations into corresponding dc voltage levels. This output voltage is converted into a digital code for further data processing. Many innovative methods have been reported in the literature for direct bridge imbalance-to-digital conversion [2]. The vast majority of techniques is based on a relaxation oscillator in which the Wheatstone bridge is one of the frequencydetermining elements.

The approach discussed in this paper is based on bridge imbalance-to-phase conversion and is employed because it enables simple analog-to-digital conversion based on counting. This feature is especially important in integrated sensors.

Manuscript received December 10, 1993; revised May 24, 1994.

The authors are with the Department of Electrical Engineering, Laboratory for Electronic Instrumentation, Delft University of Technology, 2628 CD Delft, The Netherlands.

IEEE Log Number 9406566.

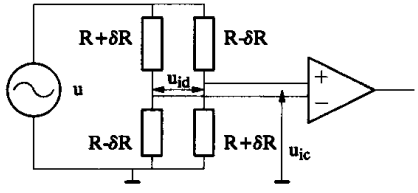

(a)

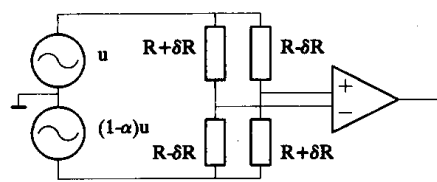

(b)

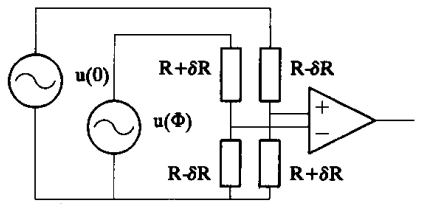

(c)

Fig. 1. (a) Standard bridge configuration. (b) Balanced drive configuration. (c) Configuration for phase readout.

The detectivity of the ac-operated bridge in the commonmode limited case (high common-mode level and low-noise) is fully dependent on the Common-Mode Rejection Ratio (CMRR $=H$ ) of the instrumentation amplifier that is traditionally implemented for the readout of the imbalance. The standard configuration is depicted schematically in Fig. 1(a). The limit of detectivity $U_{\text {id }}(\mathrm{min})$ is equal to $U_{\text {ic }} / H=U / 2 H$. The CMRR specification of the instrumentation amplifier can be relieved by a balanced drive of the bridge as shown in Fig. 1(b). The required CMRR for a limit of detectivity at $U_{\text {id }}(\min )$ can easily be shown to be limited to $H>U_{\text {ic }} / U_{\text {id }}=$ $\alpha U / 2 U_{\text {id }}(\min )$ with $\alpha$ the imbalance between the voltage sources.

Amplitude readout is performed in the conventional techniques presented in Figs. 1(a) and (b). Phase readout is possible when driving the two arms of the bridge with two separate voltages as shown in Fig. 1(c). The two voltages have an equal amplitude and a relative phase shift, $\phi$, of between $2-20^{\circ}$. The sensitivity of the output phase angle, $\theta$, on the bridge imbalance is expressed as

$$
\begin{aligned}
\theta & =-\arctan \frac{\left(1-\frac{\delta R}{R}\right) \sin \phi}{1-\cos \phi+(1+\cos \phi) \frac{\delta R}{R}} \\
& =-\arctan \frac{\phi}{2 \frac{\delta R}{R}},
\end{aligned}
$$




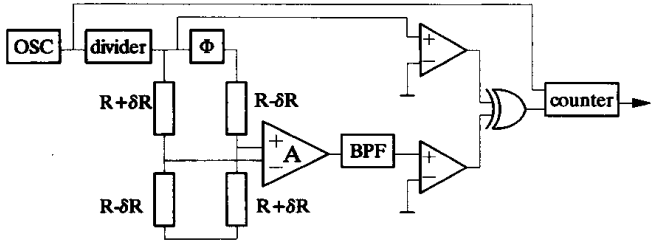

Fig. 2. Complete smart sensor system for phase operation.

for $\phi^{2} / 4<\delta R / R \ll 1$. The sensitivity, $\partial \theta / \partial \phi$, of the phase readout can be increased by selecting a small value for $\phi$, which is, however, at the expense of an increasing nonlinearity. An optimum is obtained at about $\phi=10^{\circ}[3]$.

Balancing of excitation voltages in order to relieve the CMRR requirements of the readout would make the generation of four phase-coupled signals necessary $(0, \pi, \phi$, and $\pi-\phi)$. In this work has been chosen for a relatively simple oscillator and consequently for the design of an instrumentation amplifier with high CMRR specifications.

The output phase angle depends on the matching of the amplitudes of the sinewaves and the setting of $\phi$. The main advantage of the phase output is the simple AD conversion based on counting. The phase angle can easily be converted into a duty cycle modulation, which can be used to control a counter. A relatively high bridge operating frequency is required to limit $\mathrm{AD}$ conversion time. The design presented here contains a part of the complete integrated system shown schematically in Fig. 2, which contains circuits for driving a bridge for phase operation and readout of the phase. The instrumentation amplifier and the bandpass filter have been integrated for that purpose in a bipolar process, whereas the remaining part of the system has been realized offchip.

\section{THE INSTRUMENTATION AMPLIFIER}

The essential characteristic of an instrumentation amplifier is the CMRR rather than the bandwidth or noise level. This property can be maximized using isolation and matching of the components in mainly the input stage. Isolation at lower frequencies is mainly limited by the collector-base resistance, while the degree of matching is restricted by processing tolerances. At higher frequencies the performance is determined by the magnitude and matching of the Miller capacitances. The designs reported in literature are mainly focused on differential-mode bandwidth rather than on the common-mode roll-over frequency. High common-mode rejection is maintained up to high frequencies $(100 \mathrm{kHz})$ in the design presented here.

The instrumentation amplifier is basically composed of a degenerated differential pair as an input stage [4] and a current mirror for the subtraction of the collector currents to obtain a single-ended output current. The emitter resistor ensures a linear voltage-to-current conversion at the expense of an increased noise level. The conventional degenerated input pair involves two biasing current sources as shown in Fig. 3(a) [4]-[6]. When assuming ideal npn transistors $\left(\beta_{f} \gg 1\right)$, the

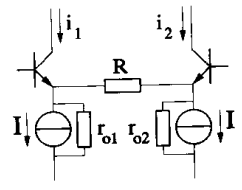

(a)

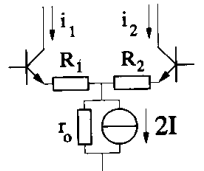

(b)
Fig. 3. (a) Conventional transconductance. (b) Improved scheme.

obtainable CMRR of this configuration is limited to

$$
H=\frac{A_{\mathrm{dd}}}{A_{\mathrm{cd}}}=\frac{r_{o}}{R \frac{\delta r_{o}}{r_{o}}} .
$$

The common-mode gain, $A_{\mathrm{cc}}$, is incorporated in the factor $F$, which is defined as

$$
F=\frac{A_{\mathrm{dd}}}{A_{\mathrm{cc}}}=\frac{r_{o}}{R} .
$$

In these equations $r_{o}$ equals the average in output resistance of the bias current sources: $r_{o}=\left(r_{o 1}+r_{o 2}\right) / 2$, and $\delta r_{o}$ equals their difference: $\delta r_{o}=\left|r_{o 1}-r_{o 2}\right|$.

Fig. 3(b) shows an improved scheme with both transistors biased by one current source. Now (2) and (3) change into

$$
H=\frac{A_{\mathrm{dd}}}{A_{\mathrm{cd}}}=\frac{2 r_{o}}{R \frac{\delta R}{R}} .
$$

and

$$
F=\frac{A_{\mathrm{dd}}}{A_{\mathrm{cc}}}=\frac{2 r_{o}}{R} .
$$

In these equations $R$ equals the sum of $R_{1}$ and $R_{2}: R=$ $R_{1}+R_{2}$, while $\delta R$ equals the difference: $\delta R=\left|R_{1}-R_{2}\right|$. The matching between two integrated resistors is usually much better than the matching of the output impedances of two current sources, especially at high frequencies. Therefore, exchanging the circuit with two biasing sources and one conversion resistor into a configuration with one current source and two matched conversion resistors results in a circuit with improved input matching and consequently in an enlarged CMRR.

To improve the linearity a second stage, consisting of $T_{3}$, $T_{4}, R_{3}$, and $R_{4}$, has been added [4]. This stage senses the input pair nonlinearity and subtracts an appropriate correction current at the output using cross coupling. The transconductance of this second stage is exactly half of the original nonlinearized transconductance. As the overall transconductance is halved, both $H$ and $F$ are decreased by a factor of 4 .

Due to the unmatched parasitic Miller capacitances between the base and the collector of the input transistors, the CMRR will decrease at high frequencies. The roll-over frequency can easily be derived from Fig. 4 . In this figure $r_{o, \text { eq }}$ equals the output impedance of the transistor parallel to the output impedance of the current source plus $R_{1}$. With $r_{\pi} \mathrm{gm}=$ $\beta_{f}$ being the current gain, the roll-over frequency equals $\left(c_{\mu}\left[r_{\pi}+r_{o, \text { eq }}\left(\beta_{f}+1\right)\right]\right)^{-1}-l / c_{\mu} \beta_{f} r_{o, \text { eq }}$. To maximize the roll-over frequency $c_{\mu}$ has to be minimized. This is realized by making the input transistors as small as possible. However, using very small transistors decreases the matching of those transistors and thus also decreases the low-frequency CMRR. 


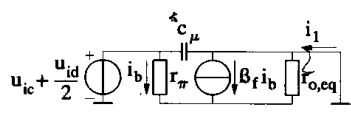

Fig. 4. Small-signal equivalent circuit of one of the input transistors.

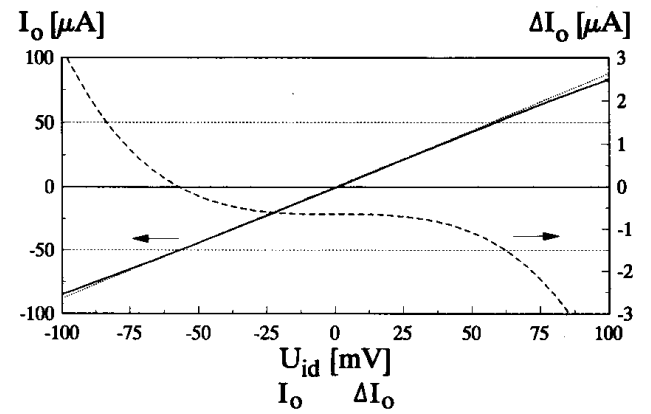

Fig. 5. Simulated nonlinearity (solid) and deviation from the best-fit line (dashed).

However, the matched degeneration resistors prevent a serious reduction of the low-frequency CMRR.

The capacitance $c_{\mu}$ depends (partly) on the base-collector voltage. Therefore, it is important to have equal loading of the two current outputs with a low impedance. This has been implemented using two pnp-current mirrors. Although the poor performance of the lateral pnp transistors gives a substantial deviation of the mirror factor from unity, only the matching of these mirrors is of importance to the CMRR. The matching of the two mirrors is improved by adding emitter resistors. The actual subtraction is performed in a Wilson npn-current mirror. The output should be connected to a small impedance, e.g., virtual ground, to have equal loading of the two pnp mirrors.

\section{Simulated PERFormanCE OF THE INSTRUMENTATION AMPLIFIER}

The behavior of the instrumentation amplifier was simulated using SPICE. Fig. 5 shows the nonlinearity up to $100 \mathrm{mV}$ differential input voltage, which is equivalent to $81 \%$ imbalance in collector current. Although the current through the mirrors is $150 \mu \mathrm{A}$, the simulated offset voltage due to nonidealities in the current mirrors is only $0.64 \mathrm{mV}$. The simulated total harmonic distortion (THD) at $100 \mathrm{kHz}$ (the bridge operating frequency) is less than $0.01 \%$ at $10 \mathrm{mV}(0.25 \%$ at $50 \mathrm{mV})$ input signal.

Fig. 6 shows the simulated amplitude response for differential- and common-mode signals. The $-3 \mathrm{~dB}$ bandwidth for DM signals is about $20 \mathrm{MHz}$, so the phase characteristic is very flat at the bridge operating frequency of $100 \mathrm{kHz}$. The bandwidth is primarily limited by the lateral pnp transistors.

The ideal simulated $\mathrm{CM}$ gain at very low frequencies is determined by the magnitude of the output impedances of the input transistors and the accuracy of the subtractor (npn mirror). The parasitic capacities are dominant at higher frequencies [6]. The simulated roll-over frequency is $14 \mathrm{kHz}$. In reality the low-frequency CM gain is dependent on 1) the magnitude and matching of the output impedances of the

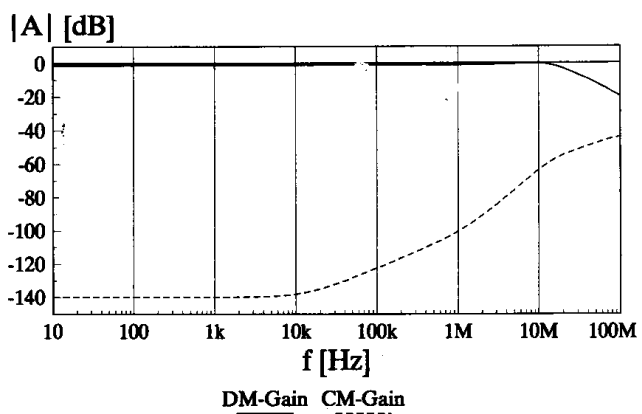

Fig. 6. Simulated differential-mode and common-mode gain.

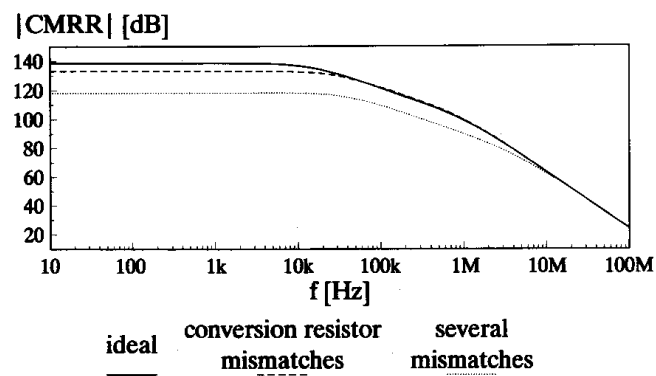

Fig. 7. Simulated Common-Mode Rejection Ratio for three matching assumptions: 1) ideal matching of all components, 2) conversion resistors matched within $1 \%, 3$ ) conversion resistors and current mirrors matched within $1 \%$ and input transistors matched within $5 \%$.

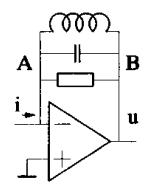

Fig. 8. Basic bandpass filter.

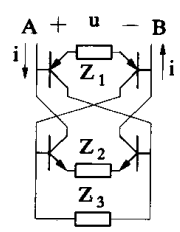

Fig. 9. Gyrator principle.

input transistors; 2) the matching of the two pnp mirrors, 3) the accuracy of the subtractor, and 4) the matching of the conversion resistors in combination with the output impedance of the current source. Taking the ratio of the DM and CM gain gives the CMRR, which is shown in Fig. 7. The solid line in this figure indicates the case of ideal CMRR with exactly matched components. The middle dashed line indicates the CMRR with $1 \%$ mismatch in the conversion resistors only. Finally, the third dotted line indicates a simulation with $1 \%$ mismatch between the conversion resistors, $5 \%$ mismatch between the emitter areas of the input transistors, and $1 \%$ 


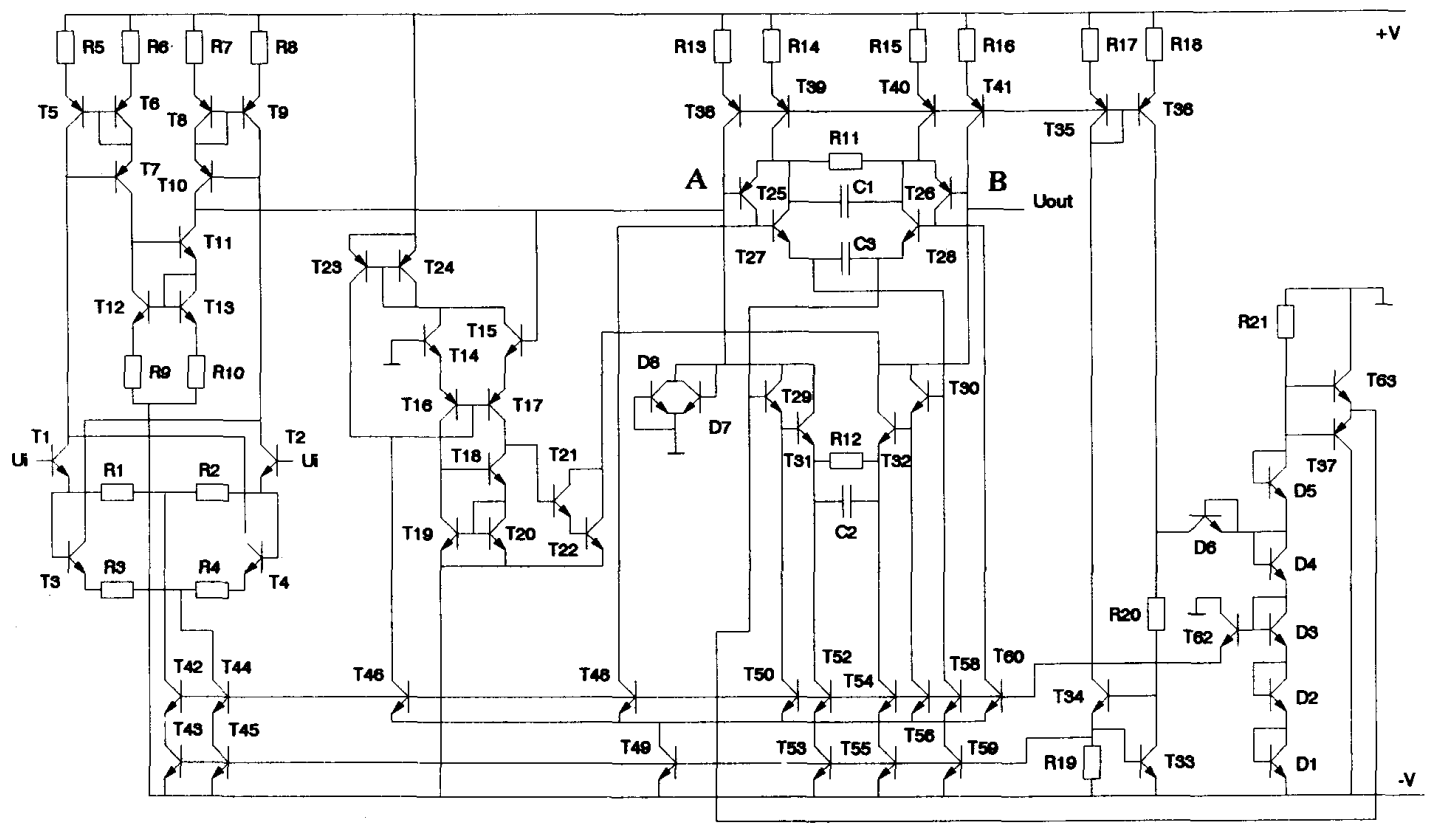

Fig. 10. Complete schematic of the instrumentation amplifier with bandpass filter.

mismatch in the three emitter resistor pairs of the three current mirrors. At $100 \mathrm{kHz}$ the simulated ideal CMRR amounts to as much as $122 \mathrm{~dB}$, which is the upper limit of the CMRR. The more realistic simulation with several mismatches gives a CMRR at that frequency of $110 \mathrm{~dB}$.

\section{THE BANDPASS FILTER}

The basic bandpass filter with a low input impedance is shown in Fig. 8. As an inductor is not integrable, the floating gyrator circuit shown in Fig. 9 has been used [7]. If $Z_{1}$ and $Z_{2}$ are composed of a resistor parallel to a capacitor and if $Z_{3}$ consists of only a capacitor, than the transfer function yields

$$
\frac{u}{i}=Z=\frac{Z_{1} Z_{2}}{Z_{3}}=\frac{G \frac{s}{Q \omega_{o}}}{1+\frac{s}{Q \omega_{o}}+\frac{S^{2}}{\omega_{o}^{2}}},
$$

where $\left.\omega_{0}=R_{11} C_{1} R_{12} C_{2}\right)^{-1 / 2}$ is the center frequency of the filter, $Q=\left(R_{11} C_{1} R_{12} C_{2}\right)^{1 / 2} /\left(R_{11} C_{1}+R_{12} C_{2}\right)$ is the quality factor, and $G=R_{11} R_{12} C_{3} /\left(R_{11} C_{1}+R_{12} C_{2}\right)$ is a gain factor.

Seven current sources are required for correct biasing of the gyrator. These currents can be derived from one reference current [8] using a pnp and an npn current mirror. The drawback of this method is the occurrence of latch-up due to the influence of current sources on each other. This can be effectively prevented by cascoding and by placing two diodes $\left(D_{7}\right.$ and $D_{8}$ in Fig. 10) antiparallel between node $A$ and ground.

\section{COMPLETE INTEGRATED CIRCUIT}

The total realized circuit is presented in Fig. 10. The noise level of this circuit at the bridge operating frequency is mainly

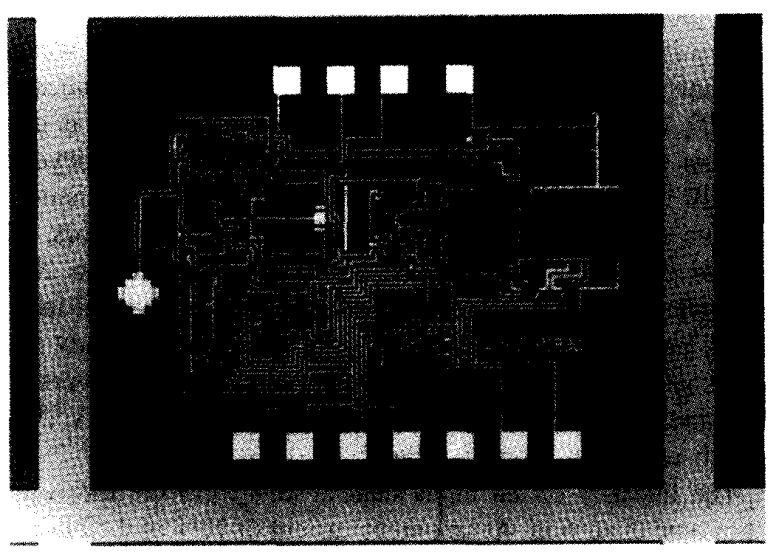

Fig. 11. Microphotograph of the integrated circuit.

determined by the conversion resistors in the input stage and the emitter resistors in the current mirrors. The simulated total equivalent input noise is about $15 \mathrm{nV} / \sqrt{ } \mathrm{Hz}$.

Fig. 11 shows a microphotograph of the circuit, which is integrated in a standard BiFET process. The instrumentation amplifier is located at the center of the upper half of the photograph. The left and the right part contain the biasing circuitry. The opamp and the gyrator circuit are found below the input stage. The capacitors of the filter were not integrated for testing purposes. The active chip area is $0.9 \times 1.7 \mathrm{~mm}^{2}$. Large-area conversion resistors have been included using a 16 $\mu \mathrm{m}$ wide boron layer with a sheet resistivity of $25 \Omega / \square$.

The amplifier and filter are tested separately. No output buffers are integrated, so at high frequencies the results are 


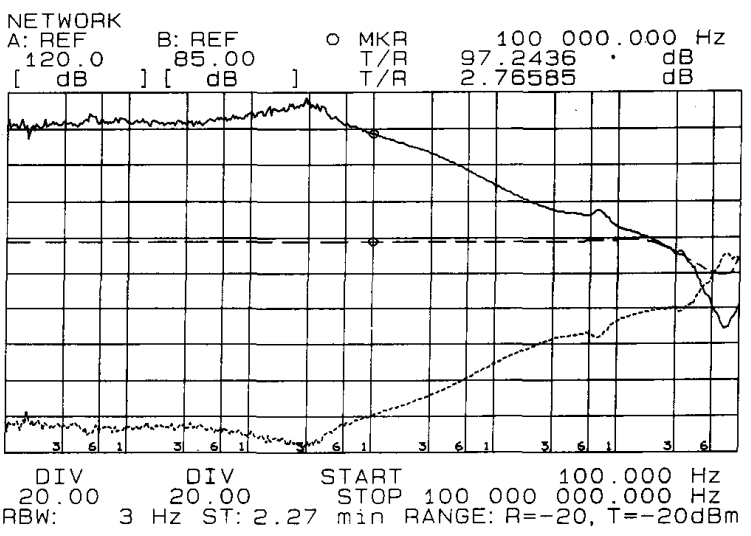

Fig. 12. Measured DM gain (dashed line, scale $B=85 \mathrm{~dB}$ max. and 20 $\mathrm{dB} /$ vert. div., $\mathrm{DM}(100 \mathrm{kHz})=2.8 \mathrm{~dB}$ ) and $\mathrm{CM}$ gain (dotted line, scale $B$, $\mathrm{CM}(100 \mathrm{kHz})=-95 \mathrm{~dB}$ ) and CMRR (solid line, scale $A=120 \mathrm{~dB} \max$. and $20 \mathrm{~dB} /$ vert.div., CMRR(100 kHz) $=97 \mathrm{~dB}$ )

slightly influenced by parasitics introduced by the measurement setup. The amplifier was loaded with a single resistor.

\section{PERFORMANCE}

The differential gain plot (dotted line) and common-mode gain plot (dashed line) of the instrumentation amplifier are shown in Fig. 12. The solid line indicates the CMRR, which amounts to $97 \mathrm{~dB}$ at the nominal bridge operating frequency of $100 \mathrm{kHz}$, which is $13 \mathrm{~dB}$ less than simulated and is due to poor component matching. A further improvement beyond $100 \mathrm{~dB}$ is possible using a better control of the sheet resistivity to give an improved matching of the conversion resistors.

The measured output noise at $30 \mathrm{kHz}$ amounts to 20.6 $\mathrm{nV} / \sqrt{ } \mathrm{Hz}$. At a DM gain of $2.8 \mathrm{~dB}$, the total equivalent input noise amounts to $15 \mathrm{nV} / \sqrt{ } \mathrm{Hz}$. As mentioned already the noise spectral power is mainly determined by the conversion resistors in the input stage and amounts to $4 \mathrm{kT} \cdot 10 \mathrm{k} \Omega \mathrm{V}^{2} / \mathrm{Hz}$, which results in $13 \mathrm{nV} / \sqrt{ } \mathrm{Hz}$.

The linearity of the input stage results from the measured harmonic distortion. At an output current of $10 \%$ of the biasing current the total harmonic distortion is $\mathrm{THD}=0.035 \%$. At high output currents (50\%) the THD is limited to $0.9 \%$.

The measured input offset voltage of the instrumentation amplifier is equal to $0.66 \mathrm{mV}$.

The gain and phase plots of the bandpass filter were measured using the Hp4195 network/spectrum analyzer, and the results are shown in Fig. 13. The zero at $1 \mathrm{kHz}$ is caused by the capacitor $C_{3}$ parallel to the output impedance of the current source. The practical filter, therefore, behaves like a bandpass filter in parallel with a lowpass filter. The dynamic range is determined by the ratio between their passband responses. Fig. 13 indicates a dynamic range equal to 30 $\mathrm{dB}$. The dynamic range can be improved up to $50 \mathrm{~dB}$ by an improved process control and by enhancing the output resistance of the current source. The limited high-frequency performance is due to processing and is compensated for by dimensioning the components that determine the resonance frequency.

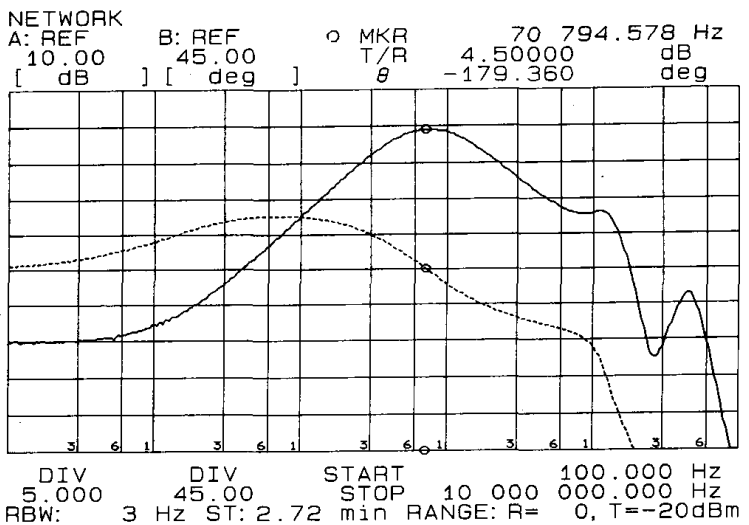

Fig. 13. Measured filter characteristics: amplitude (solid line, scale $A=10$ $\mathrm{dB}$ max. and $5 \mathrm{~dB} /$ vert.div.) and phase (dashed line, scale $B=45$ degrees max. and 45 degrees/vert.div.).

As the bridge unbalance is contained in the phase, the sensitivity of the output phase to changes in the operating frequency is of utmost importance. The measured total phase sensitivity at the center frequency of the filter amounts to only $0.60^{\circ} / \mathrm{kHz}$, so small changes in the operating frequency can be tolerated.

\section{CONCLUSION}

An instrumentation amplifier with a high common-mode rejection ratio up to $100 \mathrm{kHz}$ and a bandpass filter have been realized on a $1.5 \mathrm{~mm}^{2}$ die. Both circuits are relatively insensitive to the bipolar process parameters, so the circuits can be integrated with a silicon sensor. The circuit does not put severe demands on the frequency stability of the oscillator, because the sensitivity of the output phase for changes in the frequency amounts to only $0.6^{\circ} / \mathrm{kHz}$.

\section{ACKNOWLEDGMENT}

The authors are indebted to the Delft Institute for Micro Electronics and Sub-micron technology (DIMES) for processing the integrated circuit.

\section{REFERENCES}

[1] M. Bao, Special issue on piezoresistive sensors, Sensors and Actuators, vol. A28, pp. 81-152, 1991.

[2] J. H. Huijsing, "Signal conditioning on the sensor chip," Sensors and Actuators, vol. 10 , pp. 219-237, 1986.

[3] R. F. Wolffenbuttel and P. P. L. Regtien, "Capacitance-to-phase angle conversion for the detection of extremely small capacities," IEEE Trans. Instrum. Meas., vol. IM-36, pp. 868-872, 1987.

[4] R. A. Blauschild, "An open-loop programmable amplifier with extended frequency range," IEEE J. Solid-State Circuits, vol. SC-16, pp. 626-633, 1981.

[5] R. J. van de Plassche, "A wide-band monolithic instrumentation amplifier," IEEE J. Solid-State Circuits, vol. SC-10, pp. 424-431, 1975.

[6] A. T. van Zanten and J. H. Huijsing, "An accurate integrated voltageto-current converter," IEEE J. Solid-State Circuits, vol. SC-10, pp. 432-436, 1975.

[7] H. O. Voorman and A. Biesheuvel, "An electronic gyrator," IEEE J. Solid-State Circuits, vol. SC-7, pp. 469-474, 1972.

[8] P. R. Gray and R. G. Meyer, Analysis and Design of Analog Integrated Circuits. New York: Wiley, 1984. 


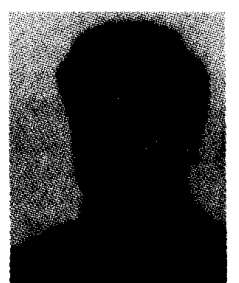

Reinoud F. Wolffenbuttel was born in Abcoude, The Netherlands, in 1958. He received the M.Sc. and $\mathrm{Ph} . \mathrm{D}$. degrees in 1984 and 1988 , respectively, both from the Delft University of Technology, Delft, The Netherlands.

In 1986 he became an assistant professor at the Laboratory for Electronic Instrumentation of The Delft University of Technology. He has been a visiting assistant professor at the the Solid-state sensors and cincuits laboratory, Department of Electrical Engineering and Computer Science, University of Michigan, Ann Arbor, where has was involved in wafer-to-wafer bonding. He is presently associate Professor at the Laboratory for Electronic Instrumentation of The Delft University of Technology and is involved in electronic instrumentation, integrated circuit design for sensor readout and fabrication compatibility of single-chip integrated data-acquisition systems in silicon.

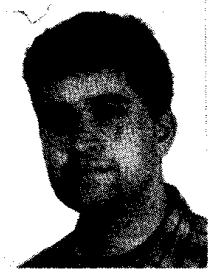

Arnold R. Schekkerman was born in Amsterdam, The Netherlands, on January 28,1968 . He received the M.Sc. degree in electrical engineering, with a specialty in aircraft avionics, from the Delft University of Technology in March 1992.

After fulfilling his military service he worked at Fokker Aircraft B.V. until the summer of 1994 at the engineering department, where he was involved in the design of avionic systems and electromagnetic compatibility. 